\title{
Breast density disclosure may do more harm than good
}

Cite as: CMAJ 2020 January 13;192:E48-9. doi: 10.1503/cmaj.1095839

Posted on cmajnews.com on December 12, 2019

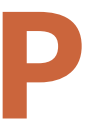

rince Edward Island is joining Nova Scotia and British Columbia in sharing breast density information directly with people having mammograms, pleasing advocates but concerning some researchers who fear the information is as likely to do harm as good.

Keeping a campaign promise, PEI Premier Dennis King announced in late November that by January 2020, all
2018. "The greater the density, the higher risk of getting breast cancer," King told the PEI legislature.

"Dense" breasts contain more connective tissue than fat. Connective tissue can mask cancers on mammograms, and breast density itself is a risk factor for breast cancer. Study results vary, but people with extremely dense breasts have about 1.5 times the risk of developing

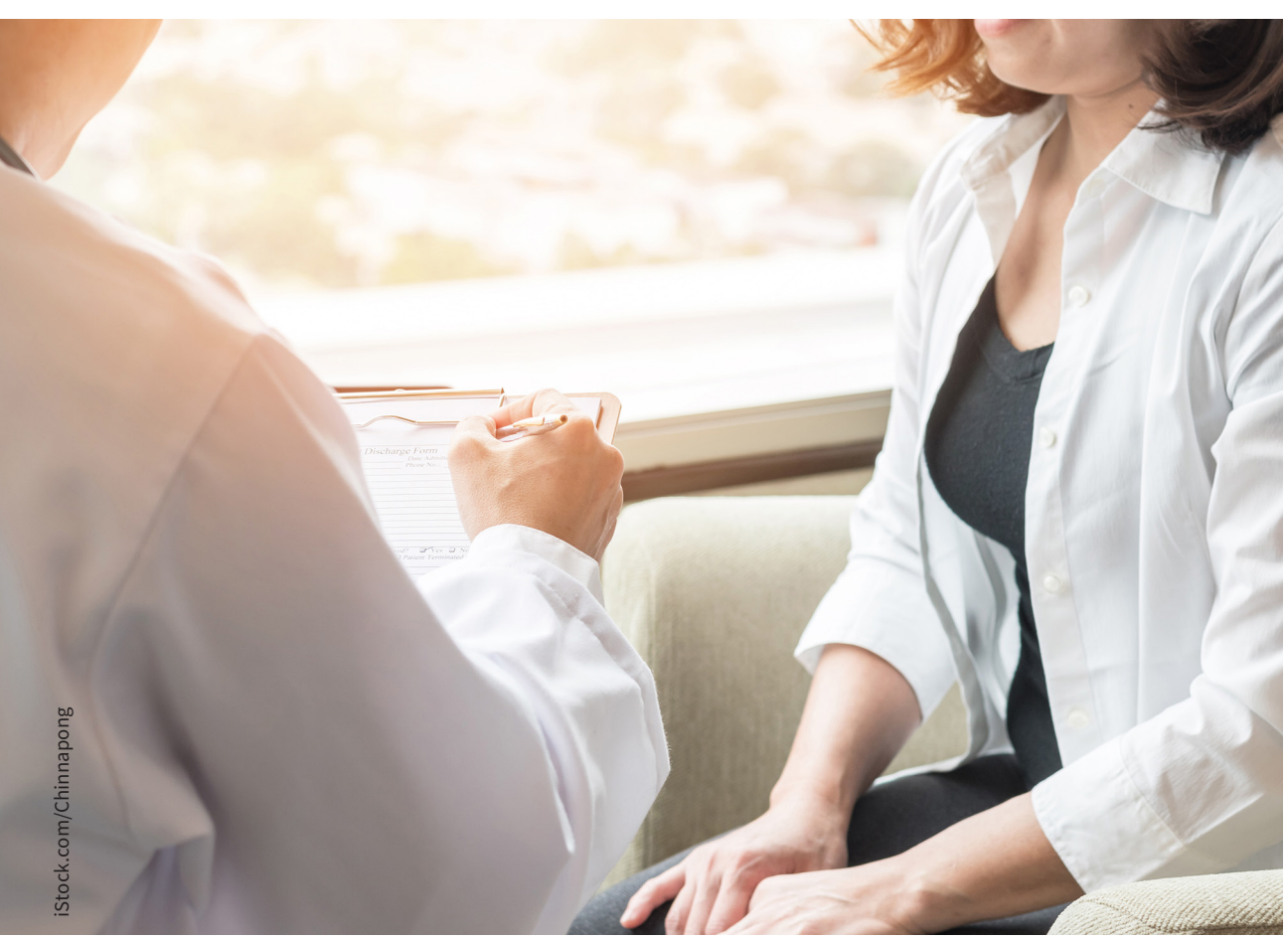

A move to share breast density information along with mammogram results may be more advocacy-based than evidence-based, some researchers warn.

Islanders having mammograms will get a cancer screening report that notifies them of their breast density. Nova Scotia began giving breast density information to mammogram recipients in October, and British Columbia has been doing so since breast cancer compared to those with average breast density.

With growing awareness of this risk, advocacy groups in the United States, and now Canada, have pushed for screening programs to disclose breast density to mammogram recipients, most of whom are women. PEl's move to disclose breast density information follows vigorous campaigning by Dense Breasts Canada. According to the group's cofounder, Jennie Dale, "we want women to be informed and to make their own decisions."

Dense Breasts Canada is also lobbying for access to ultrasounds for women in the highest density categories. "Mammograms miss over half the cancers in women in the highest density category," says Dr. Paula Gordon, a radiologist and volunteer medical advisor to Dense Breasts Canada. "It's important [to know your breast density] just like it's important for somebody to know their blood pressure."

However, other researchers question if notifying people about breast density will save lives or lead to unnecessary anxiety and testing. No research has definitively shown that giving information on breast density decreases breast cancer incidence or mortality at a population level. And medical guidelines for managing dense breasts are vague on this issue and vary depending on the source.

"In theory, it's great that women are more knowledgeable about their risk factors," says Rulla Tamimi, an epidemiologist and associate professor at Harvard University Medical School and Brigham and Women's Hospital. She coauthored a paper that found people with $50 \%$ or higher breast density are three times more likely to be diagnosed with breast cancer over a 15-year period than those with less than $10 \%$ breast density.

What people are supposed to do with breast density information is less clear. "At 
this point, I am concerned that it does more harm than good," Tamimi says. She worries that breast density results aren't accompanied by clear messaging about how to reduce the risk of breast cancer, for example, by exercising, avoiding alcohol and maintaining a healthy weight. Lacking clear guidelines, physicians are also confused about what to tell patients and how to access other screening technologies.

These concerns haven't stopped governments from mandating disclosure of breast density information. In the US, 38 states require some form of notification, but who gets notified and what they are told varies.

In Canada, Nova Scotia is the first province to use software to analyze and categorize breast density. PEI is planning to adopt a similar program. Other provinces rely on assessments by radiologists.

After a mammogram, Nova Scotia's breast screening program provides patients with a fact sheet explaining the four categories of breast density and discussing their risks. The fact sheet does not discuss ways to lower risk.

Other provinces, including Ontario, provide breast density information to family doctors or nurse practitioners. They directly notify only individuals in the highest risk category, asking them to get yearly mammograms instead of twiceyearly scans.

Sharon Batt, a health policy researcher at Dalhousie University, cautions that the move to mandatory notification isn't supported by evidence. "To me, it's advocacy- based. It's not evidence-based," Batt says. "We don't yet know whether this focus on dense breasts will save lives."

Batt is concerned that the new rules build on problematic screening policies. Only a limited subset of women benefits from early detection with mammography, which is not always accurate, she explains. Early detection may not matter with slow-growing tumours that respond to treatment, and unfortunately it also doesn't make a difference for women with fast-growing, aggressive tumours, Batt says. "The information being given is not nuanced enough. There's not focus on the inaccuracies of the screening modalities."

Laura Eggertson, Wolfville, N.S. 